\title{
$N$-acetyl-D-glucosamine kinase interacts with dynein light-chain roadblock type 1 at Golgi outposts in neuronal dendritic branch points
}

\begin{abstract}
Md Ariful Islam ${ }^{1}$, Syeda Ridita Sharif ${ }^{1}$, HyunSook Lee ${ }^{2}$, Dae-Hyun Seog ${ }^{3}$ and Il Soo Moon ${ }^{1,2}$
$\mathrm{N}$-acetylglucosamine kinase (GIcNAc kinase or NAGK) is a ubiquitously expressed enzyme in mammalian cells. Recent studies have shown that NAGK has an essential structural, non-enzymatic role in the upregulation of dendritogenesis. In this study, we conducted yeast two-hybrid screening to search for NAGK-binding proteins and found a specific interaction between NAGK and dynein light-chain roadblock type 1 (DYNLRB1). Immunocytochemistry (ICC) on hippocampal neurons using antibodies against NAGK and DYNLRB1 or dynein heavy chain showed some colocalization, which was increased by treating the live cells with a crosslinker. A proximity ligation assay (PLA) of NAGK-dynein followed by tubulin ICC showed the localization of PLA signals on microtubule fibers at dendritic branch points. NAGK-dynein PLA combined with Golgi ICC showed the colocalization of PLA signals with somal Golgi facing the apical dendrite and with Golgi outposts in dendritic branch points and distensions. NAGKGolgi PLA followed by tubulin or DYNLRB1 ICC showed that PLA signals colocalize with DYNLRB1 at dendritic branch points and at somal Golgi, indicating a tripartite interaction between NAGK, dynein and Golgi. Finally, the ectopic introduction of a small peptide derived from the C-terminal amino acids 74-96 of DYNLRB1 resulted in the stunting of hippocampal neuron dendrites in culture. Our data indicate that the NAGK-dynein-Golgi tripartite interaction at dendritic branch points functions to regulate dendritic growth and/or branching.
\end{abstract}

Experimental \& Molecular Medicine (2015) 47, e177; doi:10.1038/emm.2015.48; published online 14 August 2015

\section{INTRODUCTION}

$\mathrm{N}$-acetylglucosamine kinase (GlcNAc kinase or NAGK; EC 2.7.1.59) is a key salvage enzyme that is involved in mammalian amino-sugar metabolism. This enzyme belongs to the sugar kinase/heat shock protein 70/actin superfamily, which is defined by a fold consisting of two domains with the topology $\beta \beta \beta \alpha \beta \alpha \beta \alpha{ }^{1}$ Members of this superfamily catalyze ATP phosphoryl transfer or hydrolysis, which is coupled to a large conformational change that induces the two domains to close. ${ }^{1}$ NAGK phosphorylates GlcNAc to GlcNAc-6-phosphate in the GlcNAc recycling pathway, which leads to the formation of uridine diphosphate (UDP)-GlcNAc. This compound is then utilized to synthesize various oligosaccharide chains such as $N$-/O-glycans and glycolipids, ${ }^{2-4}$ glycosaminoglycans ${ }^{5}$ and the glycosyl phosphatidylinositol anchor of membrane-bound glycoproteins. ${ }^{6}$

Since the initial characterization of NAGK as early as $1970,{ }^{7}$ the molecular structure and properties of NAGK have been widely studied. It has been found that the molecular weights of rat and human NAGK are 37 and $39 \mathrm{kDa}$, respectively, and they form homodimers in solution. ${ }^{8}$ Human and mouse genes for NAGK have been cloned, ${ }^{9}$ and two crystal structures of homodimeric human NAGK have been described; one complexes with GlcNAc and the other with ADP and glucose. ${ }^{10}$ The $\mathrm{N}$-terminal small and C-terminal large domains have been found to form a V-shaped structure that acts as an active center for the binding of its substrates, GlcNAc and ATP. ${ }^{10}$ NAGK is a ubiquitously expressed protein, and NAGK mRNA and enzyme activity have been found in almost all tissues tested. ${ }^{9}$ The expression of NAGK has also been confirmed at the protein level in different cell lines. ${ }^{11}$ In neurons, NAGK has been found to be colocalized with microtubules (MTs) throughout the cell, with stronger intensity in the dendrites. ${ }^{12}$ The expression of NAGK in different nuclear subdomains, including speckles and paraspeckles, and around the outer nuclear membrane was described recently. ${ }^{13}$

Early in 2014, a non-canonical effect for NAGK on dendritic arborization was reported. The overexpression of NAGK

\footnotetext{
${ }^{1}$ Department of Anatomy, Dongguk Medical Institute, College of Medicine Dongguk University, Gyeongju, Republic of Korea; ${ }^{2}$ Neuroscience Section, Dongguk Medical Institute, College of Medicine Dongguk University, Gyeongju, Republic of Korea and ${ }^{3}$ Departments of Biochemistry, College of Medicine Inje University, Busan, Republic of Korea

Correspondence: Professor IS Moon, Department of Anatomy, College of Medicine, Dongguk University, 123 Dongdae-ro, Gyeongju 780-714, Republic of Korea. E-mail: moonis@dongguk.ac.kr

Received 30 October 2014; revised 23 March 2015; accepted 10 April 2015
} 
accelerated dendrite elaboration, whereas the knockdown of NAGK by short-hairpin (sh) RNA initiated the degeneration of dendrites, which could be prevented by the overexpression of exogenous NAGK. ${ }^{12}$ In a follow-up study, the same research group noted that this effect of NAGK on dendritic arborization had nothing to do with its enzymatic function, as pointmutations in NAGK that blocked its kinase activity did not affect its function on dendritogenesis; mutant NAGK was able to promote dendritogenesis as effectively as the wild-type enzyme. ${ }^{14}$ This finding indicated a structural role for NAGK in neuritogenesis, and Lee et al. ${ }^{14}$ found that the overexpression of the small, but not the large domain of NAGK resulted in dendritic degeneration. This suggests that the small domain of NAGK binds and sequesters a key component that mediates dendritogenesis.

In this study, we performed yeast two-hybrid screening to search for NAGK-interacting proteins. Among several potential candidates, dynein light-chain roadblock type 1 (DYNLRB1), a component of cytoplasmic dynein complex, draws our attention because a very similar phenomenon has been reported in neurons expressing mutant dynein. ${ }^{15,16}$ Because intracellular NAGK ${ }^{12}$ and DYNLRB1 both colocalize with MT and both are essential for dendritic growth, they are more likely to functionally interact within neuronal cells. We performed immunocytochemistry (ICC) and proximity ligation assays (PLA) and found that an interaction occurs in the cytoplasm of neuronal and non-neuronal cells and on MT fibers at dendritic branches in neurons.

\section{MATERIALS AND METHODS}

\section{Antibodies and plasmids}

The following antibodies were used at the indicated dilutions unless otherwise indicated: chicken polyclonal NAGK (1:1000 for ICC, GenWay Biotech Inc., San Diego, CA, USA, now GW22347, Sigma, St. Louis, MO, USA); mouse monoclonal NAGK (1:10 for PLA; Santa Cruz Biotechnology, Dallas, TX, USA); rabbit polyclonal NAGK (1:50 for PLA; GeneTex, Irvine, CA, USA); rabbit polyclonal DYNLRB1/LC7 (1:50 for ICC and 1:25 for PLA; Proteintech Group, Chicago, IL, USA); rabbit polyclonal dynein light-chain 1/LC8 (1:50 for PLA; Santa Cruz); rabbit polyclonal dynein heavy chain (DHC; 1:50 for ICC and 1:25 for PLA; Santa Cruz); rabbit affinity-isolated kinesin 5B (1:200 for ICC; Sigma); mouse monoclonal DDK (1:100 for PLA; Origene Technologies, Rockville, MD, USA); mouse monoclonal TGN38 (1:50; BD Biosciences, San Jose, CA, USA); rabbit polyclonal GM130 (1:25; Santa Cruz); and mouse monoclonal alpha-tubulin (1:10; broth preparation, Developmental Studies Hybridoma Bank, University of Iowa, Iowa City, IA, USA). The plasmid used for transfection was pCMV6-Myc-DDK-tagged rat NAGK (Origene).

\section{Primary culture}

Hippocampi from embryonic day 19 (E19) Sprague-Dawley rat pups were dissected, dissociated by trypsin treatment and mechanical trituration, and plated onto $12 \mathrm{~mm}$ diameter polylysine/laminincoated glass coverslips at a density of $\sim 150$ neurons $/ \mathrm{mm}^{2}$ as previously described. ${ }^{17}$ Cells were initially plated in MACS Neuro Medium (MiltenyiBiotec, Auburn, CA, USA) supplemented with MACS NeuroBrew-21, $45.95 \mu \mathrm{M}$ glutamate, $500 \mu \mathrm{M}$ glutamine, $25 \mu \mathrm{M}$ 2-mercaptoethanol and $1 \%$ penicillin-streptomycin and were fed every
4 days following plating with the same media (without additional glutamate and 2-mercaptoethanol). HEK293T cells were purchased from the Korean Cell Line Bank (Seoul, Korea) and cultured in 10\% fetal bovine serum in DMEM (Invitrogen, Grand Island, NY, USA) with $1 \%$ penicillin-streptomycin.

\section{DNA and peptide transfection}

Plasmid transfection in HEK293T cells was performed using the Genecellin transfection reagent (BioCellChallenge, Paris, France) according to the manufacturer's instructions. An 18-amino acid peptide termed 'DYNLRB1 (59-76)' and a 23-amino acid peptide termed 'DYNLRB1 (74-96)' were custom made and high-pressure liquid chromatography purified by Anygen (Gwangju, Korea) with $98 \%$ purity. These peptides are consisted of two regions of DYNLRB1 (59-76, EIDPQNDLTFLRIRSKKN and 74-96, KKNEIMVAPDKDYFLIVIQNPTE) that belong to the C-terminal part of DYNLRB1 (59-end) which interacted with NAGK in a yeast two-hybrid assay. Peptide transfection in neurons was performed using a Chariot protein transfection kit (Active Motif, Carlsbad, CA, USA) as per the manufacturer's instructions. Briefly, a peptide solution was made by mixing $250 \mathrm{ng}$ of peptide with $500 \mathrm{ng}$ of $\beta$-galactosidase in a total of $50 \mu \mathrm{l}$ of PBS, and a Chariot solution was made by diluting $2 \mu \mathrm{l}$ of the Chariot reagent in $50 \mu \mathrm{l}$ sterile $\mathrm{H}_{2} \mathrm{O}$. The peptide solution was then added to the Chariot solution and allowed to incubate for $30 \mathrm{~min}$ at room temperature (RT) to form a Chariotpeptide complex. This complex was then added to neuronal cultures (24-well plate) and immediately overlaid with $100 \mu$ l of Macs Neuro feeding medium and incubated at $37^{\circ} \mathrm{C}$ under $5 \% \mathrm{CO}_{2} / 95 \%$ air in a humidified chamber for $60 \mathrm{~min}$. An additional $100 \mu \mathrm{l}$ of feeding medium was then added, and the incubation was continued for an additional $3 \mathrm{~h}$. Then, another $200 \mu \mathrm{l}$ of same feeding medium was added and incubated for approximately $48-72 \mathrm{~h}$. The cells were then fixed and stained with a $\beta$-galactosidase staining kit (Active Motif) as per the manufacturer's protocol.

\section{Cross-linking and immunocytochemistry (ICC)}

Intracellular cross-linking was performed in live hippocampal cells with $2 \mathrm{~mm}$ dithiobis (succinimidyl) propionate (DSP, Sigma) for $30 \mathrm{~min}$ at $37^{\circ} \mathrm{C}$ inside a $5 \% \mathrm{CO}_{2}$ incubator. A $50 \mathrm{~mm}$ DSP stock solution was freshly prepared in dimethyl sulfoxide and diluted to $2 \mathrm{~mm}$ in Dulbecco's phosphate buffered saline (D-PBS; Invitrogen). The DSP solution was added directly to the neuronal cells, and the crosslinking reaction was quenched by incubating the cells in $50 \mathrm{~mm}$ Tris buffer ( $\mathrm{pH} 7.4$ ) for $15 \mathrm{~min}$ at $37^{\circ} \mathrm{C}$ inside a $5 \% \mathrm{CO}_{2}$ incubator. The cells were fixed using a sequential paraformaldehyde/methanol fixation procedure. Briefly, the cells were incubated in $4 \%$ paraformaldehyde in PBS (20 mM sodium phosphate buffer, $\mathrm{pH} 7.4$, $0.9 \% \mathrm{NaCl}$ ) at $\mathrm{RT}$ for $10 \mathrm{~min}$ followed by incubation in methanol at $-20{ }^{\circ} \mathrm{C}$ for $20 \mathrm{~min}^{18}$ ICC was performed as previously described. ${ }^{18}$

\section{Proximity ligation assay (PLA)}

Generic in situ PLA was performed using a Duolink kit (Olink Bioscience, Uppsala, Sweden) according to the manufacturer's instructions with minor modifications. Fixed cells were incubated with primary antibodies in preblocking buffer ( $5 \%$ normal goat serum, $0.05 \%$ Triton $\mathrm{X}-100$ in PBS, $\mathrm{pH} 7.4$ ) overnight at $4{ }^{\circ} \mathrm{C}$. Cells were washed three times in preblocking buffer for $20 \mathrm{~min}$ each at RT. Then, secondary antibodies conjugated with oligonucleotides, PLA probe anti-mouse MINUS and PLA probe anti-rabbit PLUS, were diluted in preblocking buffer, added to the cells and incubated for $2 \mathrm{~h}$ at $37^{\circ} \mathrm{C}$ inside a humidity chamber. Other aspects of the assay were performed 
according to the manufacturer's instructions. Briefly, cells were washed with wash buffer provided with the Duolink kit and incubated with ligation mixture and ligase for $30 \mathrm{~min}$ at $37^{\circ} \mathrm{C}$. After the hybridization and ligation of the DNA oligonucleotides, an amplification solution along with polymerase was added, which resulted in rolling circle amplification reaction. The amplified product was detected by using complimentary fluorescently labeled oligonucleotides. For the combination of PLAs and ICC, the PLA reactions were performed first followed by the addition of primary antibodies to the cells overnight at $4{ }^{\circ} \mathrm{C}$ and the incubation with fluorophoreconjugated secondary antibodies as per the ICC procedure.

\section{Image acquisition}

A Leica Research Microscope DM IRE2 equipped with I3 S, N2.1 S and Y5 filter systems (Leica Microsystems AG, Wetzlar, Germany) was used for epifluorescence microscopy. Images $(1388 \times 1039$ pixels $)$ were acquired using a high-resolution CoolSNAP CCD camera (Photometrics, Tuscon, AZ, USA) using Leica FW4000 software. Digital images were processed with Adobe Systems Photoshop 7.0 software (Adobe, San Jose, CA, USA).

\section{Yeast two-hybrid screen}

The Matchmaker LexA two-hybrid system was used for screening according to the manufacturer's protocol (Clontech Laboratories, Palo Alto, CA, USA). Briefly, the small domain of the NAGK gene (aa 1-117) was used to the DNA-BD region of the pLexA vector, and the plasmid DNA was transformed into yeast strain EGY48 carrying the $p 8 o p$-lacZ gene. Transformed EGY48 yeast strains containing the small domain of NAGK bait plasmid were transformed with a mouse brain cDNA library, ${ }^{19}$ and the cells were grown on synthetic dextrose (SD) plates supplemented with glucose but not with histidine, tryptophan or uracil (SD/-His/-Trp/-Ura). The screening of positive clones was performed on an SD/-His/-Trp/-Ura/-Leu plate containing galactose, raffinose and X-gal. Library plasmids from positive colonies were isolated and rescued using an E. coli strain (KC8 strain) on ampicillin-resistant plates. Library inserts were then amplified by PCR and analyzed by restriction enzyme digestion. Unique inserts were sequenced, and DNA and protein sequence analyses were performed using the BLAST algorithm at the National Center for Biotechnology Information. After isolation of the plasmids encoding the library clones, these plasmids were tested for interactions of the reporter gene yeast by retransformation. The activation of the reporter genes in the positive colonies was confirmed in the same experiments.

\section{Statistics}

The numbers of puncta per dendritic segment $(20 \mu \mathrm{m}, n=20)$ of typical pyramidal neurons $(n=5-10)$ from two to three independent experiments were counted and expressed as the percentage of the total (mean \pm s.d.). Data were analyzed using one-way analysis of variance with Duncan's multiple comparison post hoc tests. Statistical significance was accepted for $P$-values $<0.01$, and the analysis was conducted using SPSS version 16.0 (SPSS Inc., Chicago, IL, USA).

\section{RESULTS}

The small domain of NAGK interacts with DYNLRB1 in a yeast two-hybrid screen

Because it has been shown previously ${ }^{14}$ that the small domain of NAGK plays a critical role in dendritogenesis, we used the small domain as bait in a yeast two-hybrid screen. The NAGKinteracting proteins, which were screened at multiple times with the yeast two-hybrid system, are shown in Figure 1a. The domains of DYNLRB1 and strength of the interaction with NAGK are shown in Figure $1 \mathrm{~b}$.

\section{NAGK interacts with DYNLRB1 in primary hippocampal} neurons

Colocalization of NAGK and DYNLRB1 in ICC. To study the colocalization between NAGK and DYNLRB1, we performed ICC in rat hippocampal cultures (developmental stage IV, DIV 10) at a time of active dendritic arborization. Because the interaction between NAGK and DYNLRB1 could be transient, we added a crosslinker DSP to the culture medium for $30 \mathrm{~min}$ before fixation. The fluorescent images of neurons doublestained with antibody sets, including those against NAGK and DYNLRB1, are shown in Figure 2. Immunoreactive (IR) signals for NAGK, DYNLRB1, DHC and kinesin 5B were widely distributed throughout the soma and dendrites. Enlarged images of dendritic regions revealed many puncta, and the merged images (arrowheads) showed some colocalization of NAGK with DYNLRB1 (Figure 2a) or DHC (Figure 2b). Statistical analysis showed that the ratio of NAGK colocalization with DYNLRB1 or DHC significantly $(P<0.01)$ increased (from $12 \pm 2$ to $16 \pm 2 \%$ ) when a crosslinker was added (Figure 2d, DSP $(+)$ ). As a control experiment, NAGK/kinesin $5 \mathrm{~B}$ double-staining was performed (Figure 2c). In addition, NAGK-IR puncta colocalized with kinesin 5B-IR at a slightly lower ratio $(11 \pm 2 \%)$ than with DYNLRB1 or DHC (Figure 2d, kinesin 5B). However, the addition of DSP did not increase the ratio of colocalization, indicating that this colocalization was fortuitous due to the dense distribution of the two proteins on MT fibers. These results suggest that NAGK interacts transiently with dynein complexes.

a

NAGK-interacting protein identified by yeast two-hybrid selection

\begin{tabular}{ll}
\hline \multicolumn{1}{c}{ protein name } & $\begin{array}{c}\text { Subcellular } \\
\text { localization }\end{array}$ \\
\hline dynein light chain roadblock-type 1 (Dynlrb1) & cytoplasm \\
lactate dehydrogenase A (Ldha) & cytoplasm \\
translation elongation factor 1 alpha 1 (Eef1a1) & cytoplasm \\
\hline general transcription factor IIH, polypeptide 5(Gtf2h5) & nucleus \\
snRNP-associated polypeptideN (snRNPN) & nucleus \\
\hline
\end{tabular}

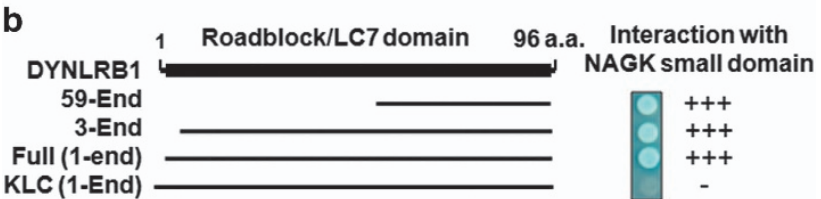

Figure $1 \mathrm{~N}$-acetylglucosamine kinase-binding proteins. (a) $\mathrm{N}$-acetylglucosamine kinase-interacting proteins identified by using the small domain as bait in yeast two-hybrid screening. (b) The interacting domains of dynein light-chain roadblock type 1 . The coding region (amino acids 1-96) of dynein light-chain roadblock type 1 is shown as a bar diagram with the conserved roadblock/LC7 domain. The two positive clones in the yeast two-hybrid selection having a coding region from 3 or 59 to the C-terminal end are also shown. Full length dynein light-chain roadblock type 1 (1-end) and kinesin light chain 1 were used as a positive and a negative control, respectively. 
Verification of NAGK-dynein complex interaction by proximity ligation assay (PLA)

We conducted in situ PLA to further confirm the direct interaction between the NAGK and dynein complexes in both neuronal and non-neuronal cells. Firstly, we applied in situ PLA for visualization of the interaction between NAGK and DYNLRB1 or DHC in primary hippocampal neurons followed by ICC with anti-tubulin antibody. In contrast to the dense distribution and a considerable amount of colocalization ratio of NAGK and dynein complex, there appeared much lower PLA signals. However, the longer the dendrites grow, the greater the number of PLA dots that are found in a single neuron. Because dynein is a motor that walks along the MT fiber, the NAGK-dynein complex would be expected to localize to MT fibers. Likewise, PLA signals for NAGK-DYNLRB1 (Figure 3a, arrowheads in upper panel) and NAGK-DHC (Figure 3a, arrowheads in the lower panel) were localized to MT fibers and were distributed in somatodendritic domains. The negative control, where primary antibody was not added, showed no PLA signals (data not shown). Because we found an unexpectedly small number of PLA signals, we performed PLA in HEK293T cells with dynein light-chain 1/LC8 (DYNLL1), another component of dynein light-chain that is positioned close to DYNLRB1 in the dynein complex. PLA interaction signals appeared throughout the cells, but again the number was low per cell (average six dots/ cell, $n=30$ ) (Figure 3b, upper panel). To further confirm the interaction, we transfected HEK293T cells with a Myc-DDKtagged NAGK plasmid and conducted PLA using a primary antibody against DDK (for exogenously expressed NAGK) coupled with an antibody against DYNLL1. PLA signals were detected throughout the cells, but the number was low as with endogenous NAGK (Figure 3b, lower panel). We also conducted PLA in hypothalamic GT1-7 neuronal cells with NAGK and DYNLRB1 primary antibodies and found PLA signals (average seven dots/cell, $n=30$, images not shown). These results clearly show an interaction between NAGK and dynein complex. a
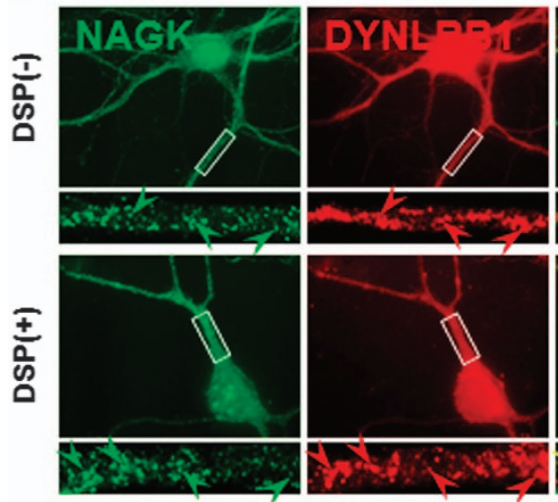

b
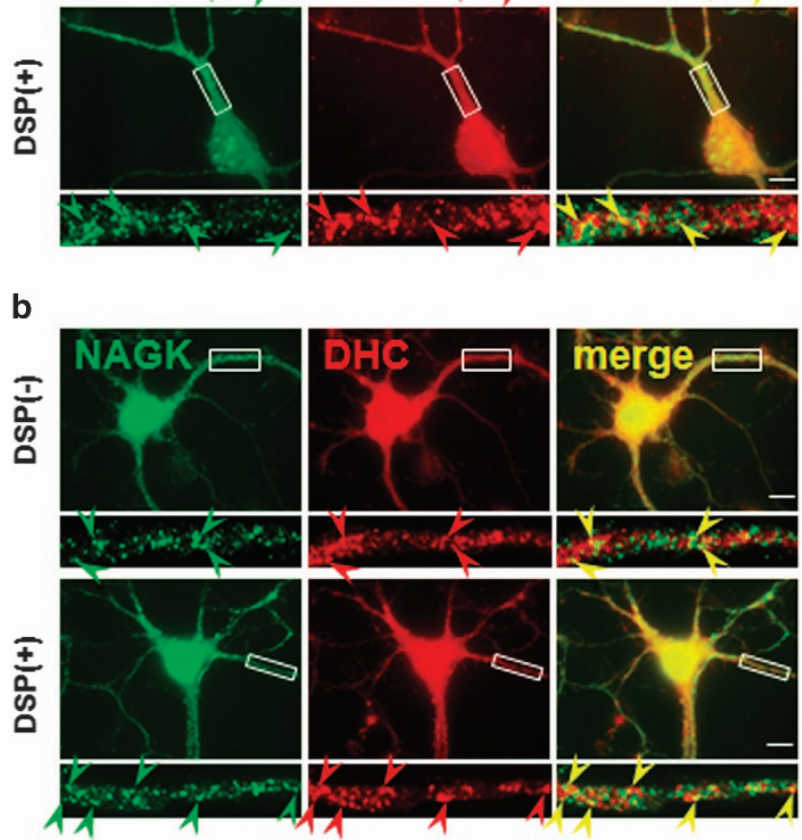

C
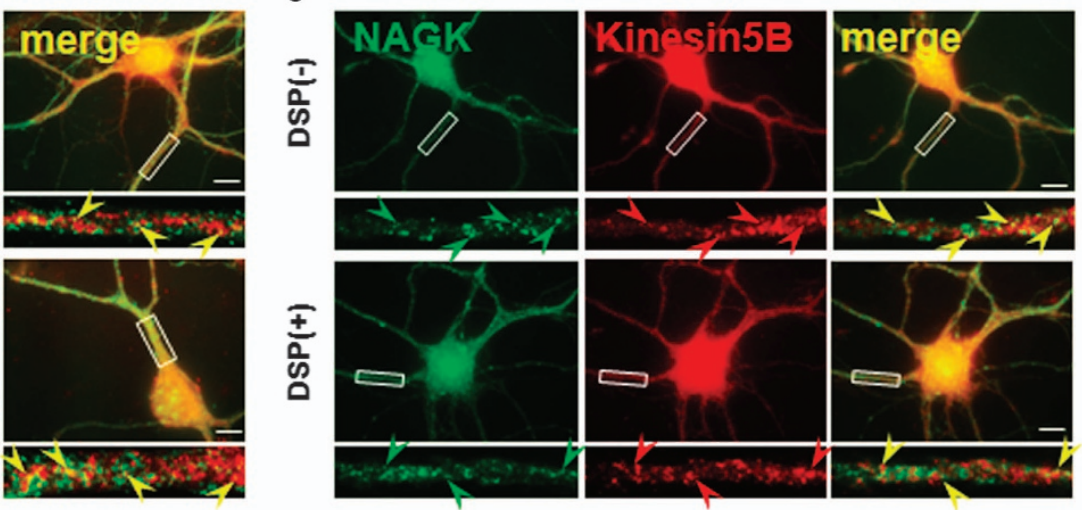

d

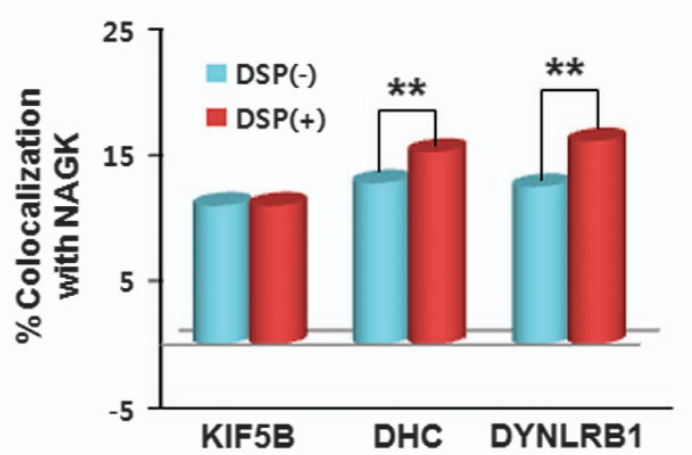

Figure 2 Immunocytochemistry showing the colocalization of $\mathrm{N}$-acetylglucosamine kinase with dynein light-chain roadblock type 1. Cultured rat hippocampal neurons were treated with dithiobis (succinimidyl) propionate (DSP+), fixed at DIV10 (stage IV) and doublestained with primary antibodies against $\mathrm{N}$-acetylglucosamine kinase and dynein light-chain roadblock type 1 (a) or dynein heavy chain (b) or kinesin 5B (c). Primary antibodies were visualized by using goat secondary antibodies conjugated to Alexa 488 ( $N$-acetylglucosamine kinase, green) or Alexa 568 (dynein light-chain roadblock type 1 or dynein heavy chain or kinesin5B, red) fluorochromes. The boxed areas of the merged images are enlarged at the bottom. Colocalized immunopuncta are indicated by arrowheads. Scale bar: $10 \mu \mathrm{m}$. Statistics (d). The numbers of puncta per dendritic segment $(20 \mu \mathrm{m}, n=20)$ were counted and expressed as a percentage of the total (mean $\pm \mathrm{s}$.d.). $* * P<0.01$. 
a
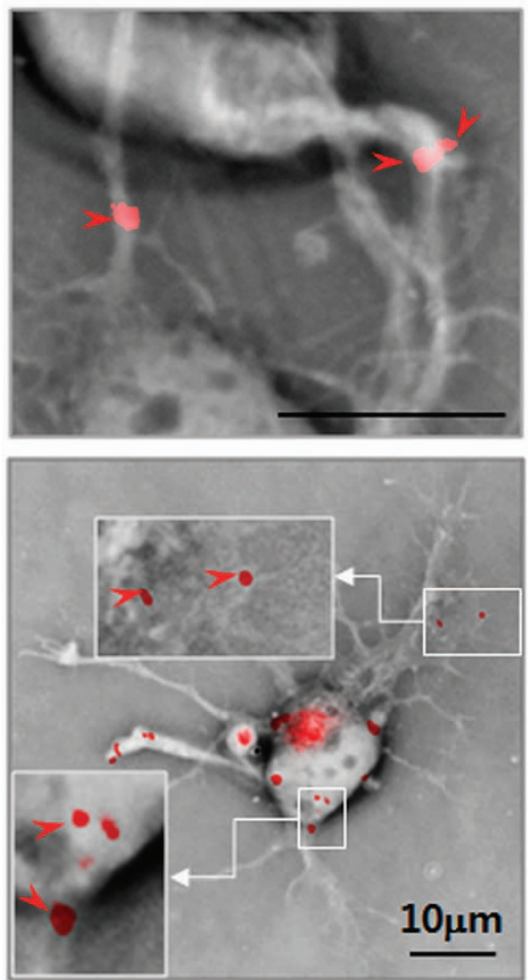
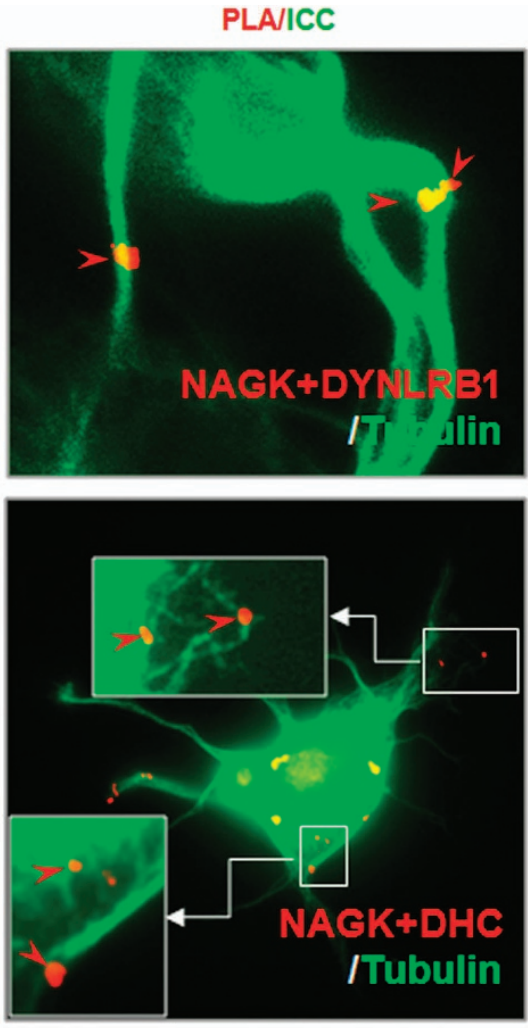

b

PLA
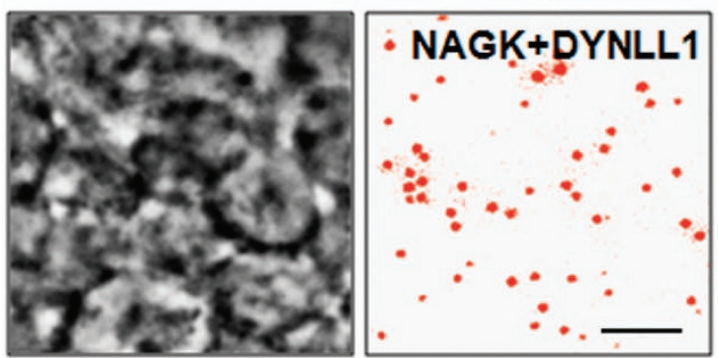

Phase/PLA
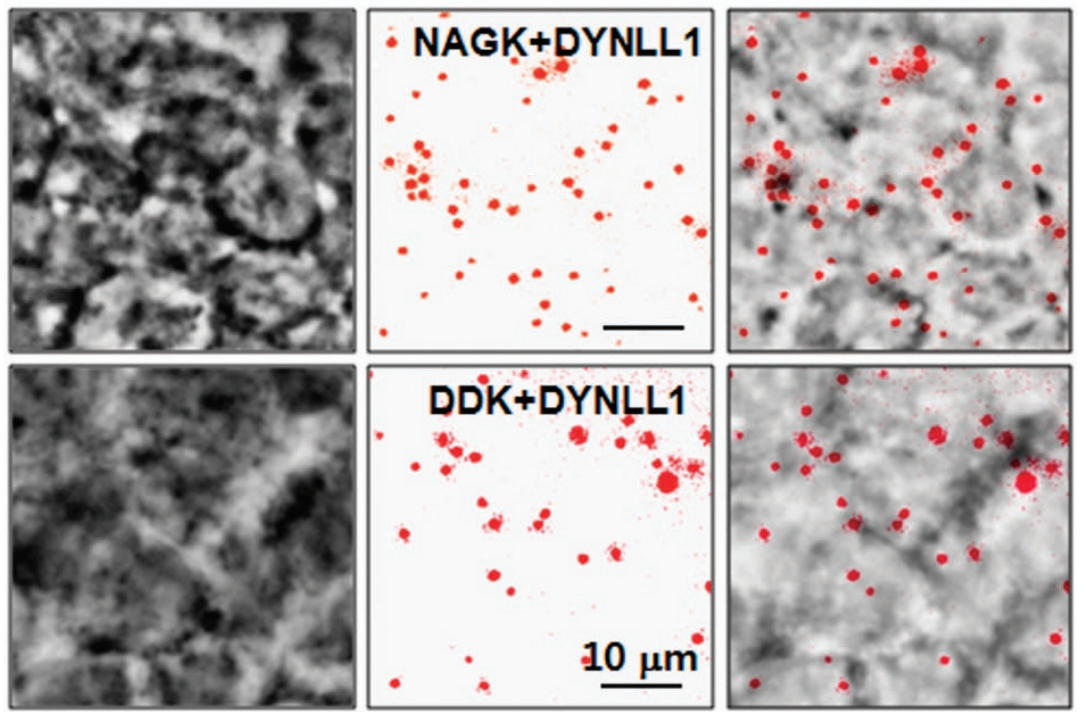

Figure 3 Proximity ligation assay showing an interaction between $\mathrm{N}$-acetylglucosamine kinase and the dynein complex. (a) Rat hippocampal neurons (DIV2) were fixed, and proximity ligation assay was performed using mouse anti- $N$-acetylglucosamine kinase and rabbit anti-dynein light-chain roadblock type 1 or anti-dynein heavy chain antibodies. The proximity ligation assay was followed by immunocytochemistry using a mouse anti-tubulin antibody (green). Proximity ligation assay/phase-contrast (Phase) and proximity ligation assay/immunocytochemistry merged images are shown. Boxed areas are enlarged (insets) to show the colocalization of the $\mathrm{N}$-acetylglucosamine kinase-dynein complex with thinner microtubule fibers (arrowheads). To obtain a better view of proximity ligation assay signals, phase-contrast images were inverted using Photoshop software. Scale bar: $10 \mu \mathrm{m}$. (b) HEK293T cells were fixed, and proximity ligation assay was performed using anti- $\mathrm{N}$-acetylglucosamine kinase and anti-dynein light-chain $1 / \mathrm{LC} 8$ antibodies (upper panel). Alternatively, the cells were transfected with Myc-DDK-tagged $\mathrm{N}$-acetylglucosamine kinase plasmids, and proximity ligation assay was conducted by using anti-DDK and anti-dynein light-chain 1/LC8 antibodies to show the interaction between exogenous $N$ acetylglucosamine kinase and dynein light-chain 1/LC8 (lower panel). Red dots represent the interaction between $\mathrm{N}$-acetylglucosamine kinase and dynein. Phase-contrast (phase), proximity ligation assay signal (red), and proximity ligation assay/phase-contrast merged images are shown. To better depict proximity ligation assay puncta, phase-contrast images were inverted using Photoshop software. Scale bar: $10 \mu \mathrm{m}$. 


\section{NAGK localizes to MT fibers}

The subcellular distribution of NAGK strongly overlaps with MTs in the neuronal somatodendritic domains. ${ }^{12}$ To show the in situ colocalization of NAGK and MT, we first performed ICC on primary hippocampal neurons. We reconfirmed the overlapping expression of NAGK and MT in mature Stage V (DIV 21) hippocampal neurons (Figure 4a, Stage V). The mature dendrites are packed with compact MT and dense NAGK, which make it impossible to see whether the NAGK puncta are localized on MTs. Therefore, we used early developmental neurons (stage II, DIV 1); in which growth cones show dispersed, separate MT fibers. Enlarged images of growth cones showed NAGK puncta and single, separate MT fibers, and the merged images showed that NAGK puncta localize to MT fibers (Figure 4a, Stage II). The statistical analysis showed that $\sim 98 \%$ of NAGK puncta $(n=30)$ were localized to MT (Figure $4 \mathrm{~b}$ ). These results strongly suggest that NAGK localizes to MT fibers, further supporting the possibility of being a part of the dynein motor complex.

\section{The interaction between NAGK and dynein frequently occurs at dendritic branch points}

Typical multipolar neurons, such as hippocampal pyramidal neurons, develop axons and dendrites in a stereotypical process that begins with a round-shaped precursor. ${ }^{20}$ The generation of new processes from soma and the formation of branches is tightly regulated and requires specific underlying molecular machinery. Because NAGK has a critical role in dendritogenesis, ${ }^{12,14}$ the positioning of NAGK-dynein complexes at neuronal subcellular sites could be important for their function. NAGK-DHC PLA was conducted on hippocampal neurons with multiple branches at the early developmental stage (stage IV), and PLA was followed by ICC with an antitubulin antibody. The merged images of PLA/phase-contrast and PLA/ICC are shown (Figure 5a). Local areas containing PLA puncta were enlarged and are shown in Figure 5b, where the localization of PLA dots at the initiation points of primary dendrites and at the initiation sites of branches is evident. We observed that some PLA dots were located at established branch joints with MTs in the neonate branch (Figure 5b, upper panel), while others missed MTs (Figure 5b, lower panel). Interestingly, NAGK-dynein PLA puncta were frequently found at the dendritic distension where MT fibers were dispersed rather than compact (Figures $5 \mathrm{a}$ and $\mathrm{b}$; dot positions 2 and 9). Statistical analysis showed that about half of the PLA puncta in dendrites were localized to branch points. Among those, $28.72 \%$ were located at an established branch joint with MTs, while $21.81 \%$ were at a branch initiation point without MTs in the neonate protrusion (Figure 5c, $n=30,30$ neurons). These phenomena suggest that the NAGK-dynein interaction has a role at dendritic branch points.

NAGK-dynein complexes colocalize with Golgi 'outposts' at dendritic branch points

The Golgi apparatus in dendritic branches, the so called Golgi 'outpost', is transported by dynein and typically localizes to
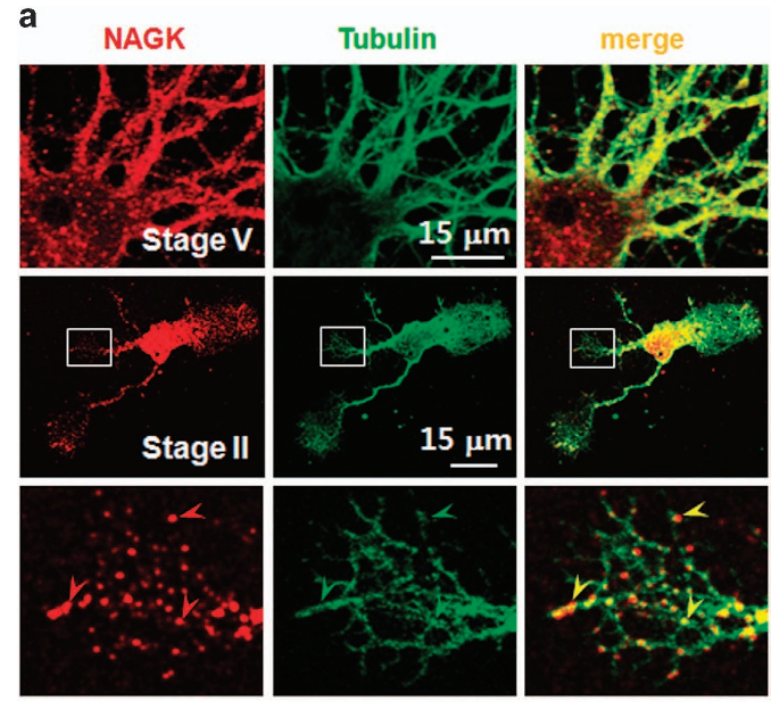

b

NAGK puncta on MT

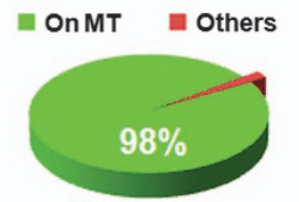

Figure 4 The colocalization of $\mathrm{N}$-acetylglucosamine kinase with tubulin confirms that $\mathrm{N}$-acetylglucosamine kinase-dynein complex locates on microtubule fibers. (a) Cultured rat hippocampal neurons at mature (stage V, DIV 21) or early developmental (stage II, DIV 1) stages were double-stained with anti- $\mathrm{N}$-acetylglucosamine kinase and anti-tubulin antibodies. Primary antibodies were visualized using goat secondary antibodies conjugated with Alexa 488 (tubulin, green) or Alexa 568 ( $\mathrm{N}$-acetylglucosamine kinase, red) fluorochromes. The right side shows the merging of two images, and a growth cone (boxed area) is enlarged at the bottom. Examples of $\mathrm{N}$-acetylglucosamine kinase puncta on microtubules are indicated by arrowheads. Scale bar: $15 \mu \mathrm{m}$. (b) Statistics. Pie chart shows that approximately $98 \%$ of $\mathrm{N}$-acetylglucosamine kinase puncta ( $n=30,3$ growth cones) were localized to microtubules.

branch bifurcation sites. We investigated whether the NAGK-dynein complex interacts with Golgi outposts to find a potential role in the transportation and/or formation of outposts. We conducted NAGK-DHC PLA to reveal the NAGK-dynein complexes, which was followed by ICC with anti-TGN38 antibody, a Golgi marker, to reveal the position of Golgi outposts. In the soma, TGN38 staining was observed as a large cluster with some small dispersed signals, whereas NAGK-dynein PLA signals were located at the somal periphery facing the dendrite joint (Figure 6a, inset 1, arrows). Along the dendritic shafts, NAGK-dynein PLA signals were found mainly at branch points and colocalized with Golgi outposts (Figure 6a, insets 2, 3, arrowheads). Golgi outposts were also colocalized with the NAGK-dynein complex at the dendritic distension (Figure 6b) where new dendritic branches were protruding (Figure 6b, arrows). 
a

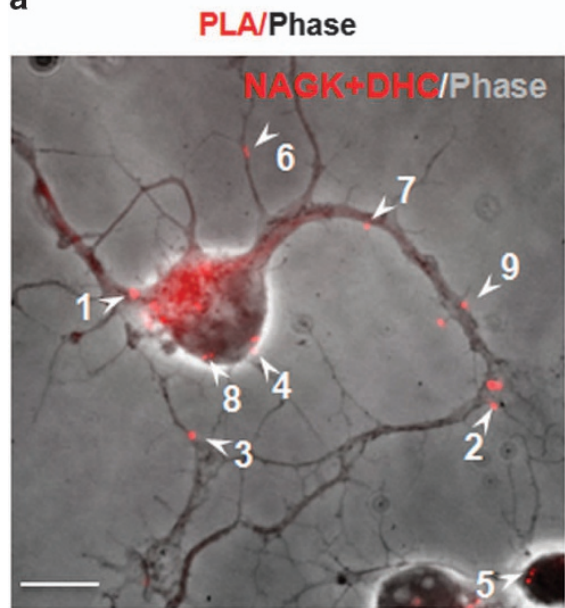

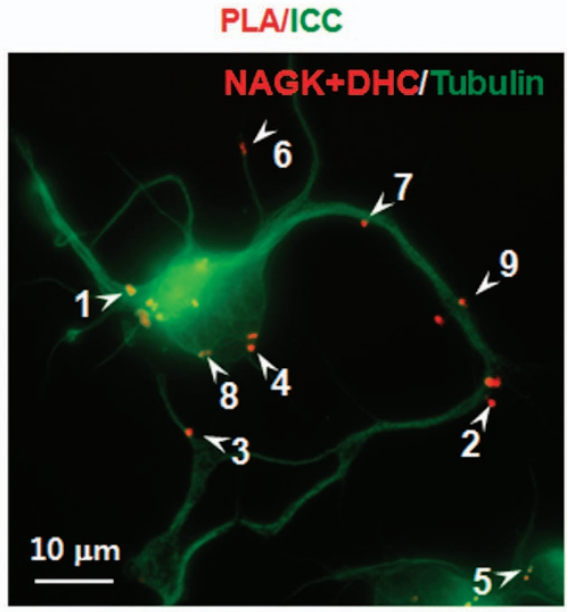

NAGK+DHC/Tubulin

b

PLA dots on branch points with MT
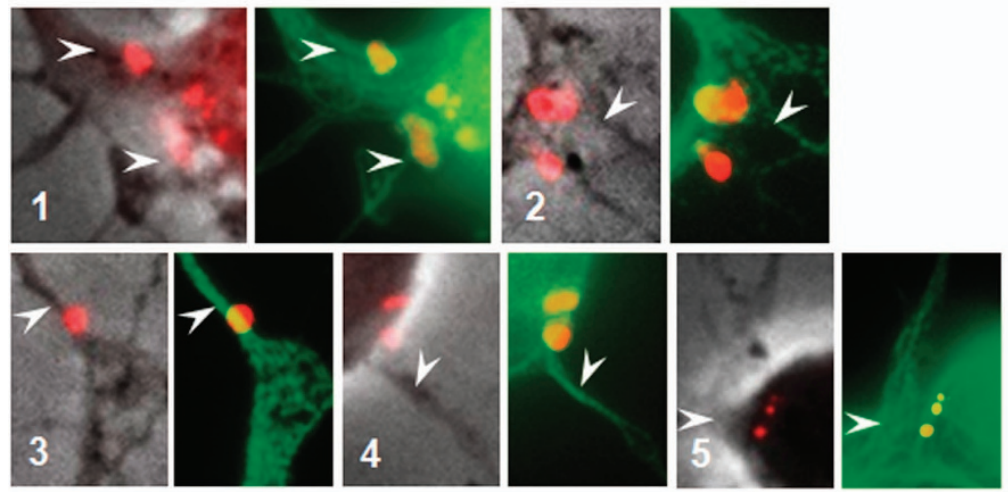

PLA dots on branch points without MT
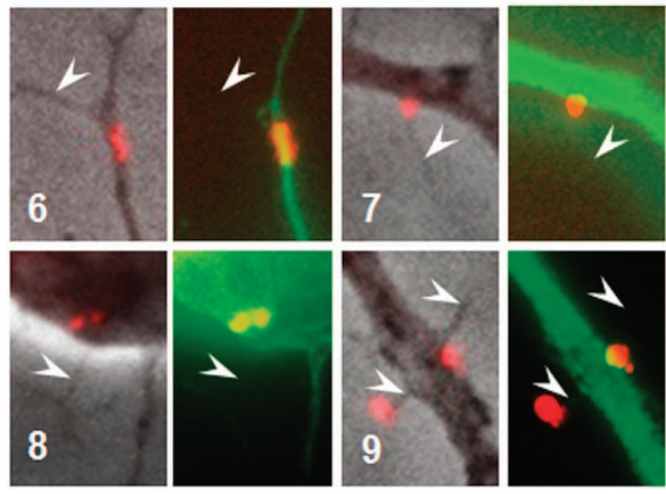

C

Positions of PLA dots
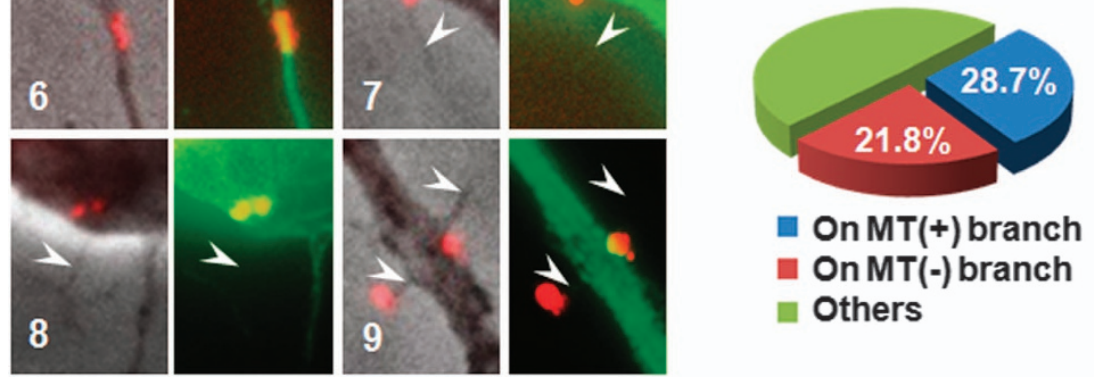

Figure $5 \mathrm{~N}$-acetylglucosamine kinase-dynein interactions occur at dendritic branch points. (a) $\mathrm{N}$-acetylglucosamine kinase-dynein heavy chain proximity ligation assay was performed in hippocampal neurons (stage IV, DIV 2) and followed by tubulin immunocytochemistry. Proximity ligation assay/phase-contrast (phase) and proximity ligation assay/immunocytochemistry merged images are shown. The proximity ligation assay puncta (red) are indicated by arrowheads and with numbers. Scale bar: $10 \mu \mathrm{m}$. (b) Local areas of proximity ligation assay puncta are enlarged. Proximity ligation assay puncta were sorted into two categories: one with 'established' branch joints where the microtubule fiber is already developed (upper panel) and the other at branch initiation sites where the microtubule is yet to develop (lower panel). Positions of branches are indicated with arrowheads. (c) Statistics. Percentages of $\mathrm{N}$-acetylglucosamine kinase-dynein heavy chain proximity ligation assay puncta at branch joints with or without microtubule in neonate protrusions are shown by a pie chart. $n=30,30$ neurons.

Colocalization of NAGK-Golgi complexes with DYNLRB1 on MTs at dendritic branch points

To further strengthen the evidence for NAGK-dynein-Golgi colocalization on MT fibers at dendritic branch points, we conducted NAGK-TGN38 PLA followed by ICC with an anti- tubulin antibody in stage IV hippocampal neurons that have many proximal dendritic branch bifurcation points (Figure 7a). In addition to in the soma, NAGK-TGN38 PLA signals (red dots marked by arrowheads) were also found in dendrites, and ICC with an anti-tubulin antibody further showed that the PLA 
signals localize to MT fibers at dendritic branch points (Figure 7a, insets 1 and 2). Having shown the NAGK-Golgi interaction on MT fibers, we next investigated the tripartite NAGK-GolgiDYNLRB1 interaction. To do so, NAGK-GM130 PLA was followed by anti-DYNLRB1 ICC. NAGK-GM130 PLA signals showed a similar distribution to those from NAGK-TGN38 PLA, including at the somal area toward the base of the apical dendrites (Figure 7b, box i) and at the dendritic branch points (Figure 7b, box ii). Interestingly, the NAGK-GM130 PLA puncta mostly colocalized with DYNLRB1 ICC labeling (Figure $7 \mathrm{~b}$, insets 1-3). The statistical analysis showed that $\sim 99 \%$ of the NAGK-TGN38 PLA signals $(n=30)$ were localized to MTs and $96 \%$ of NAGK-GM130 PLA puncta $(n=30)$ colocalized with DYNLRB1 (Figure 7c). Together, these results strongly support a three-way interaction of NAGK-dynein-Golgi outpost at dendritic branch points.

\section{Ectopic introduction of peptides derived from DYNLRB1} induce dendritic degeneration

To confirm the NAGK-DYNLRB1 interaction and its effect on dendritogenesis, we designed two small peptides, DYNLRB1 (59-76) (EIDPQNDLTFLRIRSKKN) and DYNLRB1 (74-96)

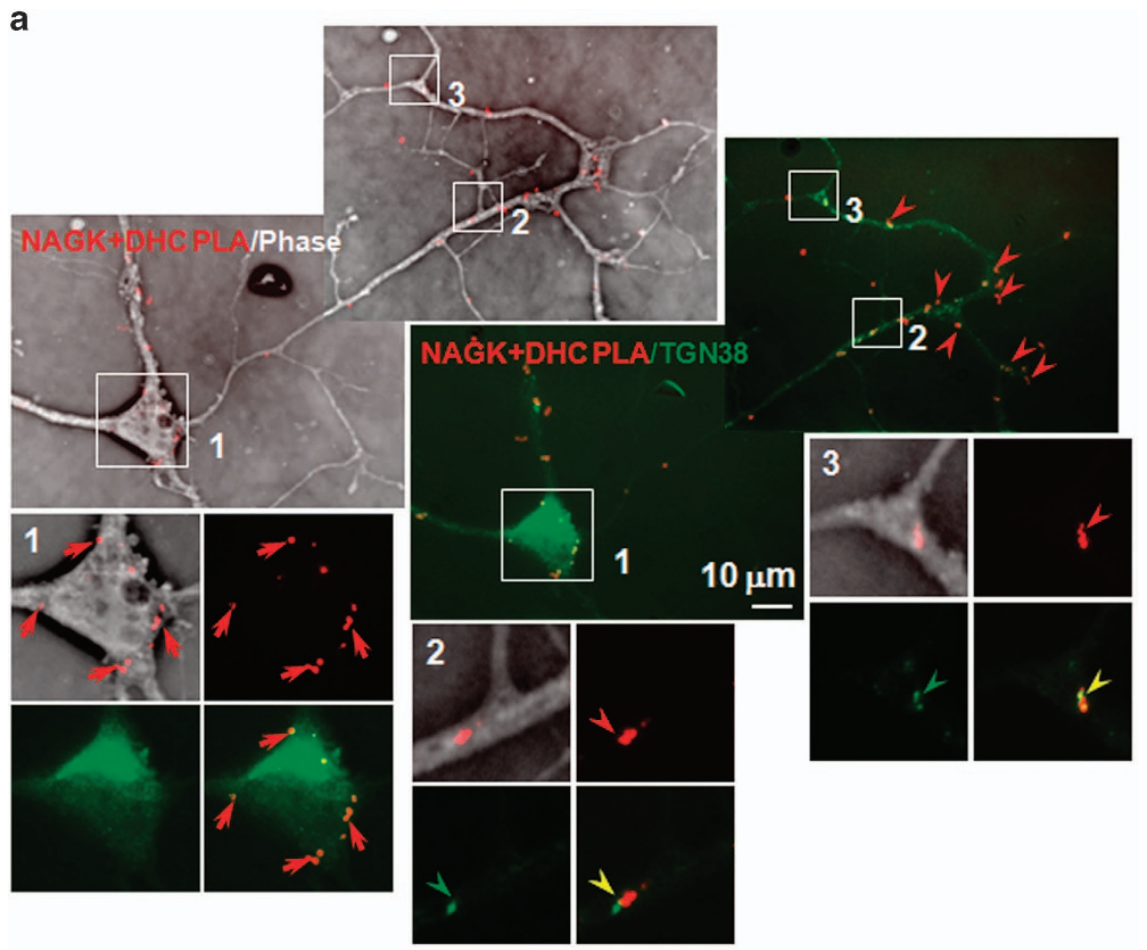

b

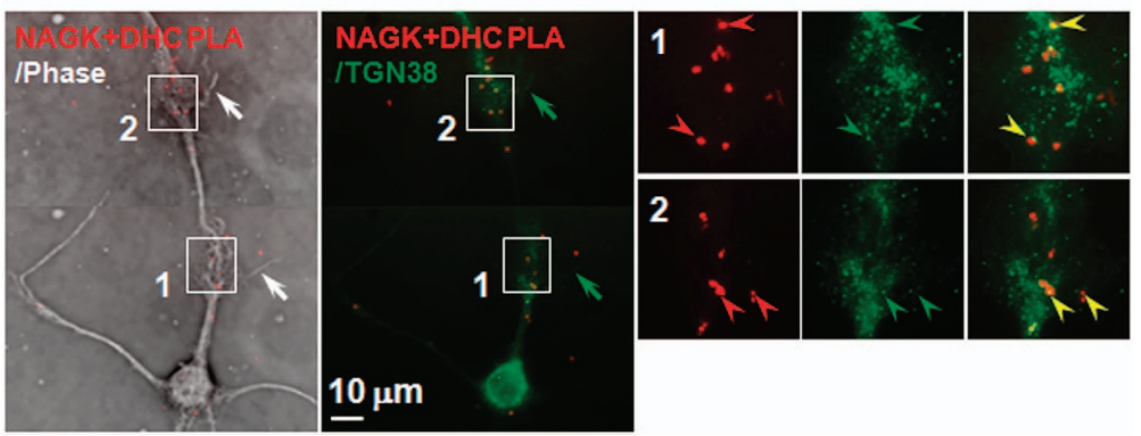

Figure 6 Colocalization of $\mathrm{N}$-acetylglucosamine kinase-dynein complexes with Golgi outposts on dendritic branch points. $\mathrm{N}$-acetylglucosamine kinase-dynein heavy chain proximity ligation assay was performed in hippocampal neurons (developmental stage IV) and was followed by immunocytochemistry with an anti-TGN38 antibody to mark Golgi particles. Merged images of NAGK-DHC PLA/phase ( $N$-acetylglucosamine kinase+dynein heavy chain proximity ligation assay/phase contrast) and NAGK-DHC PLA/immunocytochemistry are shown. (a) Proximity ligation assay signals at soma (boxed area 1) and distal branch points (boxed areas 2, 3) are enlarged to show the colocalization of $\mathrm{N}$-acetylglucosamine kinase-dynein complex (red) with Golgi particles (green) oriented to the soma-dendrite joint (inset 1 , arrows) or with the Golgi outpost (green) at distal branching sites (inset 2, 3, arrowheads). (b) A high frequency of proximity ligation assay puncta (red) and Golgi outposts (green) was found at dendritic distensions. Small branches protruding out of the distensions are marked with arrows. Dendritic distensions (boxed areas 1,2 ) are enlarged (insets) to show the colocalization of $\mathrm{N}$-acetylglucosamine kinase-dynein complex (red) with Golgi particles (green) marked by arrowheads. Scale bar: $10 \mu \mathrm{m}$. 


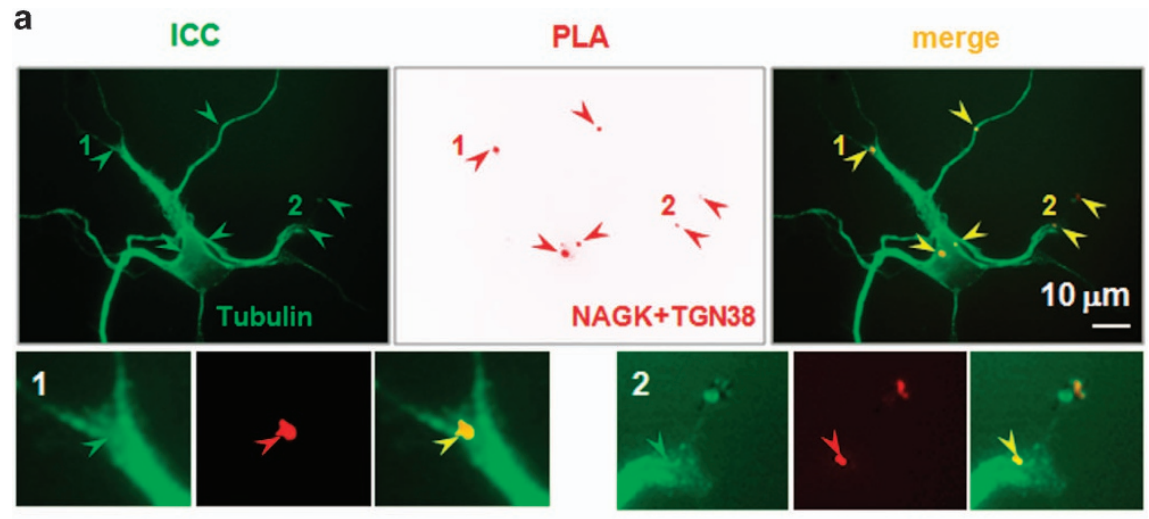

b

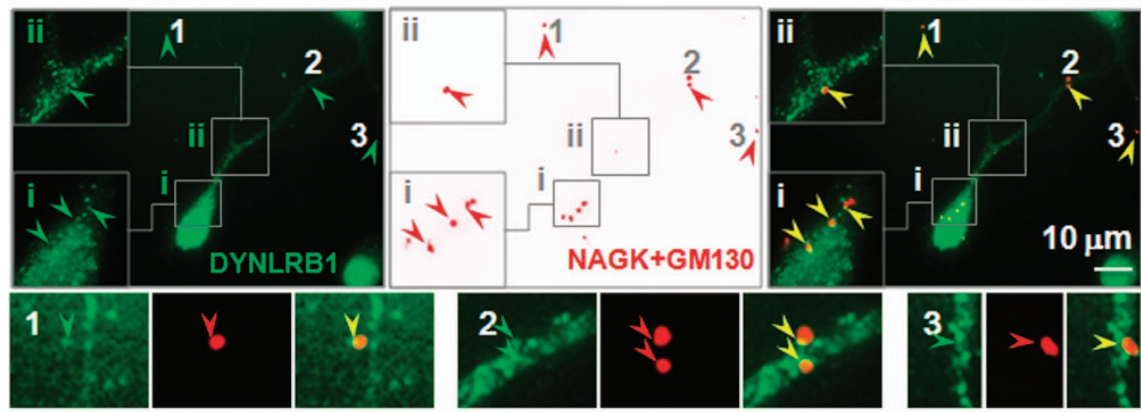

c

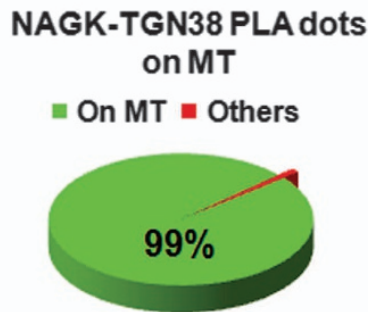

\begin{abstract}
NAGK-GM130 PLA dots ON DYNLRB1
\end{abstract}

" On DYNLRB1 $=$ Others

Figure 7 Co-localization of $\mathrm{N}$-acetylglucosamine kinase-Golgi complexes with dynein light-chain roadblock type 1 on microtubule at dendritic branch points. (a) $\mathrm{N}$-acetylglucosamine kinase-TGN38 proximity ligation assay (red dots) was followed by immunocytochemistry (green) with an anti-tubulin antibody. Images for proximity ligation assay signals are inverted to better show proximity ligation assay puncta (marked with red arrowheads). The positions of proximity ligation assay signals at branch points are marked with numbers and enlarged in insets. Scale bar: $10 \mu \mathrm{m}$. (b) $\mathrm{N}$-acetylglucosamine kinase-GM130 proximity ligation assay was followed by immunocytochemistry with an anti-dynein light-chain roadblock type 1 antibody. The proximity ligation assay puncta in the soma (box i) were enlarged to show that proximity ligation assay signals facing the apical dendrite at the somal Golgi apparatus merged with dynein light-chain roadblock type 1 signals. Proximity ligation assay dot positions (red) in dendrites are enlarged for better visualization (box ii, 1-3). Scale bar: $10 \mu \mathrm{m}$. (c) Statistics. Pie charts show that $\sim 99 \%(n=30)$ of $N$-acetylglucosamine kinase-TGN38 proximity ligation assay dots were localized to microtubules and $\sim 96 \%(n=30)$ of $N$-acetylglucosamine kinase-GM130 proximity ligation assay signals were colocalized with dynein light-chain roadblock type 1.

(KKNEIMVAPDKDYFLIVIQNPTE), from the C-terminal half of DYNLRB1, which binds to the NAGK small domain in our yeast two-hybrid analysis (Figure $1 \mathrm{~b}$; clone 59 -end). We co-transfected each of the peptides coupled with $\beta$-galactosidase into stage IV neurons and identified the transfected neurons by color development using $\beta$-galactosidase. We found that neurons transfected with the C-terminal end peptide (DYNLRB1 (74-96)) but not DYNLRB1 (59-76) (Figure 8a, left image) displayed shorter, stunted dendrites (Figure $8 \mathrm{a}$, right image, arrows). In both cases, neurons had long and apparently intact axons (Figure 8a, arrowhead), which confirmed that the DYNLRB1 (74-96) peptide resulted in dendritic but not axonal degeneration. Sholl analysis ${ }^{21}$ showed that neurons transfected with DYNLRB1 (74-96) had significantly fewer dendritic crossing points $(P<0.01 ; n=30$ transfected neurons) at all distances, except at $10 \mu \mathrm{m}$ (Figure 8b). This phenomenon is very similar to the phenotype that results from NAGK knockdown or the dominant-negative expression of its small domain. ${ }^{12,14}$ These results confirm that NAGK interacts with the C-terminal end domain of DYNLRB1 and that the interruption of this interaction results in the degeneration of dendritic arborizations. 

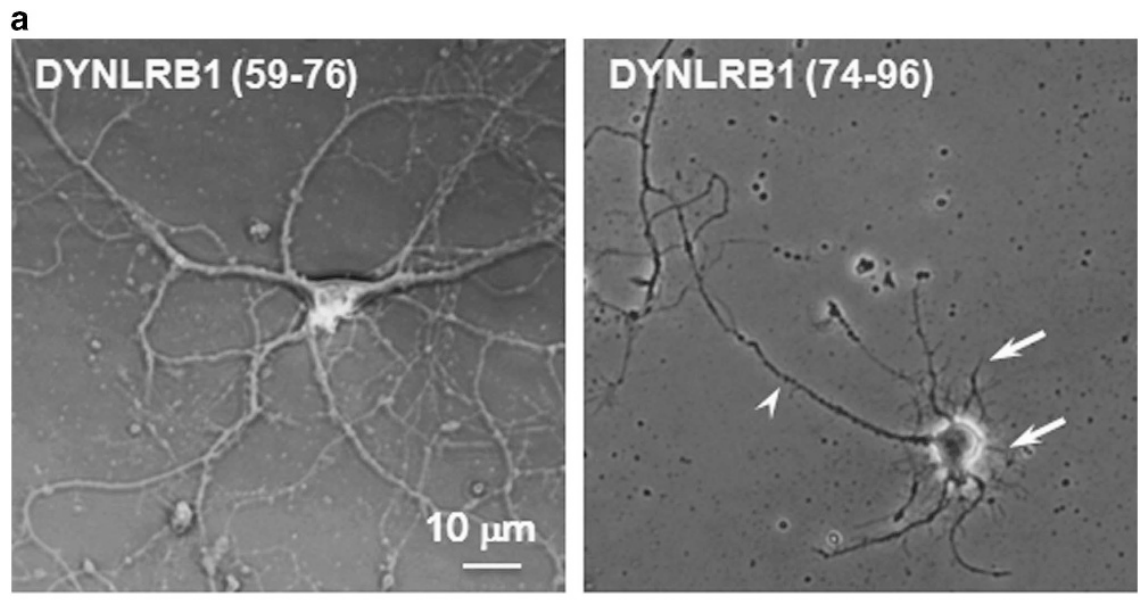

b

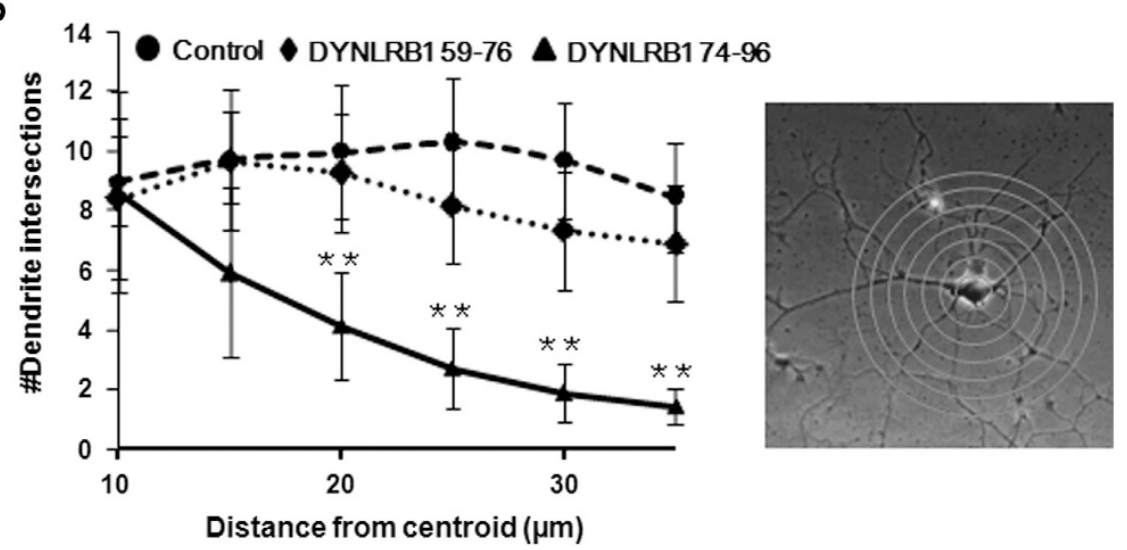

Figure 8 Transfection with a small peptide from dynein light-chain roadblock type 1 induced dendritic degeneration. (a) Co-transfection of either DYNLRB1 (59-76) or DYNLRB1 (74-96) peptide with $\beta$-galactosidase was performed and was stained with $\beta$-galactosidase staining kit. Neurons transfected with DYNLRB1 (59-76) peptide showed healthy dendrites. In contrast, neurons transfected with DYNLRB1 (7496) peptide showed short, stunted dendrites (arrows), while the axon (arrowhead) was apparently unaffected. Scale bar: $10 \mu \mathrm{m}$. (b) Sholl analysis. The numbers of dendritic intersections were counted at distances of 10,15,20, 25 and $30 \mu \mathrm{m}$ (concentric circles; see the right image for an example) from soma centroids of neurons (30 neurons in each category) transfected with $\beta$-galactosidase only (control), with DYNLRB1 (59-76) or with DYNLRB1 (74-96). ${ }^{*} P<0.01$.

\section{DISCUSSION}

In this study, we found by yeast two-hybrid screening that a hexosamine kinase, NAGK, interacts with DYNLRB1. ICC images show that NAGK and DYNLRB1 formed dense puncta in neuronal dendrites, and the merging of the two ICC images revealed a partial colocalization of the two proteins; however, the ratio of colocalization was low $(\sim 12 \%)$. Intracellular crosslinking by DSP, which covalently crosslinks interacting proteins, resulted in a small but statistically significant increase (by $\sim 4 \%$ ) in the colocalization ratio, suggesting a proteinprotein interaction between the two proteins. The interaction was confirmed by PLA, which showed that NAGK-DYNLRB1 binding occurred on MT fibers at dendritic branch points. We also showed that the NAGK-dynein complex could be found at proximal and distal branch points that contained Golgi outposts. Finally, the ectopic inclusion of a small peptide derived from the NAGK-binding domain of DYNLRB1 resulted in dendritic degeneration, confirming an essential role for the NAGK-DYNLRB1 interaction in dendritogenesis.

Every protein interaction is assumed to be an integrated part of a larger network and to be critical for cellular signal transduction processes. NAGK is an essential enzyme for amino sugar metabolism where it is involved in the salvage pathway for GlcNAc recycling. ${ }^{22}$ Recently, it was revealed that NAGK regulates cellular processes involved in neuronal dendritogenesis. ${ }^{12,14}$ This effect on dendritic development was independent of its kinase activity, with the small domain having a critical role. ${ }^{14}$ The discovery of this non-canonical function for NAGK prompted us to search for binding partners of the small domain. We performed yeast two-hybrid screening using the small domain of NAGK as bait and found that NAGK interacts with DYNLRB1. Cytoplasmic dynein is a large, multisubunit protein complex that functions in transport towards the (-) end of MTs. The cytoplasmic dynein complex consists of two heavy chains, two intermediate chains, four light 
intermediate chains and light chains of the LC7/roadblock, LC8 and the Tctex1/rp3 protein families. Based on the latest nomenclature of cytoplasmic dynein, ${ }^{23}$ DYNLRB1 is a member of the LC7/roadblock protein family. The two highly homologous mammalian roadblocks, DYNLRB1 and DYNLRB2, have been shown to be expressed in a variety of tissues. In contrast to the generally high level of DYNLRB2 expression, DYNLRB1 is relatively expressed more strongly in the brain, heart, liver and pancreas, ${ }^{24}$ suggesting a differential role in these tissues. Indeed, Drosophilla melanogaster LC7-null mutants exhibit phenotypes including defects in dendrite growth, axonal transport and neuroblast cell division. ${ }^{25}$

DYNLRB1 acts as one of several non-catalytic accessory components that could link dynein to cargos and to adapter proteins that regulate dynein function. DYNLRB1 has been reported to have multiple non-dynein interaction partners including the Rab6 family of GTPase regulators, ${ }^{26}$ human reduced folate carrier ${ }^{27}$ and the transforming growth factor- $\beta$ receptor complex..$^{28,29}$ In the structure of DYNLRB1, there is a small hole formed by residues $68,69,79-81$ and $88-90$ from three $\beta$ sheets $(\beta 3, \beta 4$ and $\beta 5)$, into which a residue can fit. ${ }^{30}$ This surface has a positive electric charge, which has been suggested to be the potential site for protein interaction by means of a salt bridge. Our present study supports the hypothesis that this surface may be the site to which NAGK binds. First, our yeast two-hybrid assay showed that the 59-96 amino acid region, which encompasses this hole, interacts with the small domain of NAGK. Second, the exogenous introduction of the DYNLRB1 (74-96) peptide, but not DYNLRB1 (59-76), resulted in neurons with shorter dendrites. Interestingly, neurons transfected with the DYNLRB1 (74-96) peptide had apparently undisturbed axons. The shortened dendrites and apparently intact axons are characteristic features that are very similar to the morphology observed following NAGK knockdown. ${ }^{12}$ Thus, our results indicate that the amino acids 74-96 of DYNLRB1 are responsible for binding to the small domain of NAGK and that this interaction is essential for dendritogenesis.

The question remains as to how NAGK, as part of the dynein complex, regulates dendritic arborization. Eukaryotic cells use cytoskeletal motor proteins to transport many different intracellular cargos. Two different mechanisms have been evolved to cope with the diverse cargos that are transported on MTs. Numerous kinesins have evolved to facilitate positive-end transport on MTs. In contrast, a single cytoplasmic dynein serves the minus end-directed transport for similarly diverse cargos. This could be achieved by employing adaptors that link dynein to diverse cargos (see review by Kardon and Vale). ${ }^{31}$ In this respect, the frequent localization of NAGK-dynein PLA puncta at dendritic branch points drew our attention because its distribution profile is very similar to that of Golgi outposts in dendrites. In mammalian neurons, the Golgi apparatus is present as Golgi stacks in the cell body and discrete Golgi outposts in the branch points of dendrites. ${ }^{32-35}$ It is also known that small Golgi particles move from the soma to dendrites via dynein and that proteins such as Drosophila golgin Lava lamp ${ }^{36}$ and Golgin $160^{37}$ link Golgi to dynein for this movement. In this study, we have shown a tripartite interaction of NAGK-dyneinGolgi on the MTs in both the soma and dendritic branch points, suggesting that NAGK has a role in the regulation of Golgi transport by the dynein motor. The involvement of the NAGK-DYNLRB1 complex in Golgi trafficking is further supported by the fact that DYNLRB1 colocalizes with Rab6 GTPase at the Golgi apparatus in Neuro-2A cells. ${ }^{26}$ The localization of $\mathrm{DHC}^{38}$ and Tctex- ${ }^{39}$ at Golgi bodies has also been revealed. Thus, the three-way interaction between dynein, NAGK and Golgi on MTs at dendritic branch points supports a non-canonical function for NAGK in Golgi transport.

This raises a question as to the function of NAGK-dynein complex at a dendritic branch points. During early neuronal development in culture, MT fibers are dispersed in the distended regions of dendritic shafts. ${ }^{40}$ In this study, we observed that these expansion sites are enriched with the NAGK-dynein complex and Golgi outposts. Ye et al. ${ }^{41}$ reported that the growth of dendrites requires membrane trafficking through the classical secretory pathway via the Golgi apparatus. In addition, that study also showed that the directional movements of dendritic Golgi outposts correlate with the extension and retraction of dendritic branches. These results provide strong evidence that local dendritic branching and growth is controlled, in part, by the dynamics and abundance of dendritic Golgi outposts. ${ }^{32}$ Supporting the role for Golgi outposts in dendritic branching, Ori-McKenney et al. ${ }^{42}$ reported that Golgi outposts were instrumental in dendritic morphogenesis by functioning as sites for acentrosomal MT nucleation. Our study shows that NAGK-dynein complex frequently localizes to neonate protrusions with or without MTs. The association of NAGK-dynein complexes with Golgi outposts suggests that the complex has a role in directional Golgi dynamics and MT nucleation toward the neonate protrusions. Our findings shed light on the interactions of NAGK with dynein and Golgi outposts and their roles in dendritic growth. Further investigation is needed into the molecular mechanisms underlying these interactions.

\section{CONFLICT OF INTEREST}

The authors declare no conflict of interest.

\section{ACKNOWLEDGEMENTS}

We thank Eun-jung Jung for technical assistance. This research was supported by the Basic Science Research Program through the National Research Foundation of Korea (NRF), funded by the Ministry of Education, Science and Technology

(NRF-2012R1A1A2006116).

1 Hurley $\mathrm{JH}$. The sugar kinase/heat shock protein 70/actin superfamily: implications of conserved structure for mechanism. Annu Rev Biophys Biomol Struct 1996; 25: 137-162.

2 Hakomori S. Traveling for the glycosphingolipid path. Glycoconj J 2000; 17: 627-647.

3 Schachter $\mathrm{H}$. The joys of HexNAc. The synthesis and function of $\mathrm{N}$ - and O-glycan branches. Glycoconj J 2000; 17: 465-483. 
4 Van den Steen P, Rudd PM, Dwek RA, Opdenakker G. Concepts and principles of O-linked glycosylation. Crit Rev Biochem Mol Biol 1998; 33: 151-208.

5 Esko JD, Lindahl U. Molecular diversity of heparin sulfate. J Clin Invest 2001; 108: 169-173.

6 Watanabe R, Murakami Y, Marmor MD, Inoue N, Maeda Y, Hino J et al. Initial enzyme for glycosylphosphatidylinositol biosynthesis requires PIG-P and is regulated by DPM2. EMBO J 2000; 19: 4402-4411.

7 Datta A. Studies on hog spleen $\mathrm{N}$-acetylglucosamine kinase. I. Purification and properties of $\mathrm{N}$-acetylglucosamine kinase. Biochim Biophys Acta 1970; 220: 51-60.

8 Hinderlich S, Nöhring S, Weise C, Franke P, Stäsche R, Reutter W. Purification and characterization of $\mathrm{N}$-acetylglucosamine kinase from rat liver: comparison with UDP- $\mathrm{N}$-acetylglucosamine 2-epimerase/ $\mathrm{N}$ acetylmannosamine kinase. Eur J Biochem 1998; 252: 133-139.

9 Hinderlich S, Berger M, Schwarzkopf M, Effertz K, Reutter W. Molecular cloning and characterization of murine and human $\mathrm{N}$-acetylglucosamine kinase. Eur J Biochem 2000; 267: 3301-3308.

10 Weihofen WA, Berger M, Chen H, Saenger W, Hinderlich S. Structures of human $\mathrm{N}$-acetylglucosamine kinase in two complexes with $\mathrm{N}$-acetylglucosamine and with ADP/glucose: insights into substrate specificity and regulation. J Mol Biol 2006; 364: 388-399.

11 Ligos JM, de Lera TL, Hinderlich S, Guinea B, Sanchez L, Roca R et al. Functional interaction between the Ser/Thr kinase PKL12 and N-acetylglucosamine kinase, a prominent enzyme implicated in the salvage pathway for GIcNAc recycling. J Biol Chem 2002; 277: 6333-6343.

12 Lee HS, Cho SJ, Moon IS. The non-canonical effect of $\mathrm{N}$-acetyl-Dglucosamine kinase on the formation of neuronal dendrites. Mol Cells 2014; 37: 248-256.

13 Sharif SR, Lee HS, Islam MA, Seog DH, Moon IS. N-Acetyl-D-Glucosamine Kinase is a component of nuclear speckles and paraspeckles. Mol Cells 2015; 38: 402-408.

14 Lee HS, Dutta S, Moon IS. Upregulation of dendritic arborization by $\mathrm{N}$-acetyl- $D$-glucosamine kinase is not dependent on its kinase activity. Mol Cells 2014; 37: 322-329.

15 Sato D, Tsuyama T, Saito M, Ohkura H, Rolls MM, Ishikawa F et al. Spatial control of branching within dendritic arbors by dynein-dependent transport of Rab5-endosomes. Nat Cell Biol 2008; 10: 1164-1171.

16 Zheng Y, Wildonger J, Ye B, Zhang Y, Kita A, Younger SH et al. Dynein is required for polarized dendritic transport and uniform microtubule orientation in axons. Nat Cell Biol 2008; 10: 1172-1180.

17 Brewer GJ, Torricelli JR, Evege EK, Price PJ. Optimized survival of hippocampal neurons in B27-supplemented neurobasal, a new serumfree medium combination. J Neurosci Res 1993; 35: 567-576.

18 Moon IS, Cho SJ, Jin I, Walikonis R. A simple method for combined fluorescence in situ hybridization and immunocytochemistry. Mol Cells 2007; 24: 76-82.

19 Takeda S, Yamazaki H, Seog DH, Kanai Y, Terada S, Hirokawa N. Kinesin superfamily protein 3 (KIF3) motor transports fodrin-associating vesicles important for neurite building. J Cell Biol 2000; 148: 1255-1265.

20 Dotti CG, Sullivan CA, Banker GA. The establishment of polarity by hippocampal neurons in culture. J Neurosci 1988; 8: 1454-1468.

21 Sholl DA. Dendritic organization in the neurons of the visual and motor cortices of the cat. J Anat 1953; 87: 387-406.

22 Berger M, Chen H, Reutter W, Hinderlich S. Structure and function of $\mathrm{N}$-acetylglucosamine kinase. Identification of two active site cysteines. Eur J Biochem 2002; 269: 4212-4218.

23 Pfister KK, Fisher EM, Gibbons IR, Hays TS, Holzbaur EL, Mclntosh JR et al. Cytoplasmic dynein nomenclature. J Cell Biol 2005; 171: 411-413.

24 Jiang J, Yu L, Huang X, Chen X, Li D, Zhang Y et al. Identification of two novel human dynein light chain genes, DNLC2A and DNLC2B, and their expression changes in hepatocellular carcinoma tissues from 68 Chinese patients. Gene 2001; 281: 103-113.
25 Reuter JE, Nardine TM, Penton A, Billuart P, Scott EK, Usui T et al. A mosaic genetic screen for genes necessary for Drosophila mushroom body neuronal morphogenesis. Development 2003; 130: 1203-1213.

26 Wanschers B, Van de Vorstenbosch R, Wijers M, Wieringa B, King SM, Fransen $\mathrm{J}$ et al. Rab6 family proteins interact with the dynein light chain protein DYNLRB1. Cell Motil Cytoskeleton 2008; 65: 183-196.

27 Ashokkumar B, Nabokina SM, Ma TY, Said HM. Identification of dynein light chain road block-1 as a novel interaction partner with the human reduced folate carrier. Am J Physiol Gastrointest Liver Physiol 2009; 297 G480-G487.

28 Tang $Q$, Staub CM, Gao G, Jin Q, Wang Z, Ding W et al. A nove transforming growth factor- $\beta$ receptor-interacting protein that is also a light chain of the motor protein dynein. Mol Biol Cell 2002; 13: 4484-4496.

29 Jin Q, Gao G, Mulder KM. Requirement of a dynein light chain in TGF $\beta /$ Smad3 signaling. J Cell Physiol 2009; 221: 707-715.

30 Liu JF, Wang ZX, Wang XQ, Tang Q, An XM, Gui LL et al. Crystal structure of human dynein light chain Dnlc2A: structural insights into the interaction with IC74. Biochem Biophys Res Commun 2006; 349: 1125-1129.

31 Kardon JR, Vale RD. Regulators of the cytoplasmic dynein motor. Nat Rev Mol Cell Biol 2009; 10: 854-865.

32 Horton AC, Racz B, Monson EE, Lin AL, Weinberg RJ, Ehlers MD. Polarized secretory trafficking directs cargo for asymmetric dendrite growth and morphogenesis. Neuron 2005; 48: 757-771.

33 Horton AC, Ehlers MD. Dual modes of endoplasmic reticulum-to-Golgi transport in dendrites revealed by live-cell imaging. J Neurosci 2003; 23 6188-6199.

34 Gardiol A, Racca C, Triller A. Dendritic and postsynaptic protein synthetic machinery. J Neurosci 1999; 19: 168-179.

35 Pierce JP, Mayer T, McCarthy JB. Evidence for a satellite secretory pathway in neuronal dendritic spines. Curr Biol 2001; 11: 351-355.

36 Papoulas 0 , Hays TS, Sisson JC. The golgin Lava lamp mediates dyneinbased Golgi movements during Drosophila cellularization. Nat Cell Biol 2005; 7: 612-618.

37 Yadav S, Puthenveedu MA, Linstedt AD. Golgin160 recruits the dynein motor to position the Golgi apparatus. Dev Cell 2012; 23: 153-165.

38 Roghi C, Allan VJ, Wall JS, Brown JC. Dynamic association of cytoplasmic dynein heavy chain la with the Golgi apparatus and intermediate compartment. J Cell Sci 1999; 112: 4673-4685.

39 Tai AW, Chuang JZ, Sung CH. Localization of Tctex-1, a cytoplasmic dynein light chain, to the Golgi apparatus and evidence for dynein complex heterogeneity. J Biol Chem 1998; 273: 19639-19649.

40 Dailey ME, Smith SJ. The dynamics of dendritic structure in developing hippocampal slices. J Neurosci 1996; 16: 2983-2994.

41 Ye B, Zhang Y, Song W, Younger SH, Jan LY, Jan YN. Growing dendrites and axons differ in their reliance on the secretory pathway. Cell 2007; 130: 717-729.

42 Ori-McKenney KM, Jan LY, Jan YN. Golgi outposts shape dendrite morphology by functioning as sites of acentrosomal microtubule nucleation in neurons. Neuron 2012; 76: 921-930.

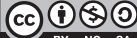

This work is licensed under a Creative Commons Attribution-NonCommercial-ShareAlike 4.0 International License. The images or other third party material in this article are included in the article's Creative Commons license, unless indicated otherwise in the credit line; if the material is not included under the Creative Commons license, users will need to obtain permission from the license holder to reproduce the material. To view a copy of this license, visit http://creativecommons.org/licenses/by-nc-sa/4.0/ 